\title{
Is Rat-tail a new reference model for implant osseointegration studies?
}

Matthieu Renaud, Manon Nerzic, Olivier Fesquet, Serge Soliveres, Philippe Bousquet.

UFR Odontologie Montpellier, EA 4203, Laboratoire bio-santé et nanoscience,

Université Montpellier

\section{Abstract}

Animal studies are necessary to precede clinical studies. Recently the rat tail model has been proposed as a model for studying bone regeneration with easily surgical approach, effective control of postoperative pain and a decrease of animal number. The aim of this study is to widen the rat tail model indication to implant osseointegration and bone implant contact calculation.

Wistar rats were used in this experimentation. Adapted impacted-type of titanium implants were inserted through tail vertebrae. At the end of each experimentation period, the rats were sacrificed. X-ray microtomography (Micro-CT) and histology were used to visualize bone formation and to calculate bone implant contact. Micro-CT showed osseointegrated implants in caudal vertebra with the possibility to obtain bone implant-contact.

The results suggest that the rat caudal vertebrae may serve as a good preclinical model for studying and following implant osseointegration with the possibility of multiple testing within the same experimental animal.

\section{Background and Aim}

Rat models are suitable to assess bone regeneration providing sufficient statistical significance. X-ray micro-computed tomography (Micro-CT) is a powerful tool to assess bone healing. Micro-CT also allows determining bone density.

Recently we have proposed a new rat model using caudal vertebrae. This model can be used to study osseointegration. Live animals can be easily monitored by micro-CT to investigate for bone implant contact and bone formation in the vicinity of implants.

The aim of this study is to use the rat-tail vertebrae model for bone implant contact and implant osseointegration studies. This model permits to exploit different vertebrae in the same experiment decreasing the number of animal and, respecting the UE directive. First, adapted impacted-implants were placed into the vertebra. Then, micro-ct measurements were realized to establish BIC and to validate this new animal approach.

\section{Methods and Materials}

The experimental work was performed on the wistar rats with weight parameters from 380 to $450 \mathrm{~g}$. Critical size defect drillings were performed through the center of the vertebrae. Impacted-type titanium $\left(\mathrm{Ti}_{6} \mathrm{Al}_{4} \mathrm{~V}\right)$ implants were placed. At the end of the experimentation period, the rats were sacrificed by intraperitoneal injection of Pentothal with a suitable dosage. After sacrifice, the tails were amputated between C1-C2 vertebrae. The soft tissues were carefully removed mechanically and the samples were fixed.

Implanted samples were scanned and measurements were made on the Region of Interest on the computer-reconstructed 3D samples. The $\mathrm{BIC}$ analysis was performed with CTanalyser software. Then, the pieces were dehydrated and embedded in epoxy resin blocks. Transverse sections were stained with toluidine blue.

\section{Results}

Micro-CT scanning showed the implant position through the vertebra. Vertebra reconstruction shows presence of bone around implant three month after the surgical procedure. Bone implant contact was calculated from these data and them gray scale pixels to obtain the quantity and the percentage of bone implant contact. Bone implant contact (BIC), percentage of BIC along the threads of the implant surface; and bone volume fraction / density; percentage of the periimplant bone volume to the total could be calculated. Percent of bone implant contact is obtained dividing total intersection surface by tissue surface. Calculation of BIC represents 54.98 +/- 5 percent.
The histology analysis confirmed this osseointegration and showed bone-implant contact by a lot of trabecular bone in contact with titanium three months after implantation.

Micro-CT and histology analyses showed osseointegration by bone implant contact after three months.
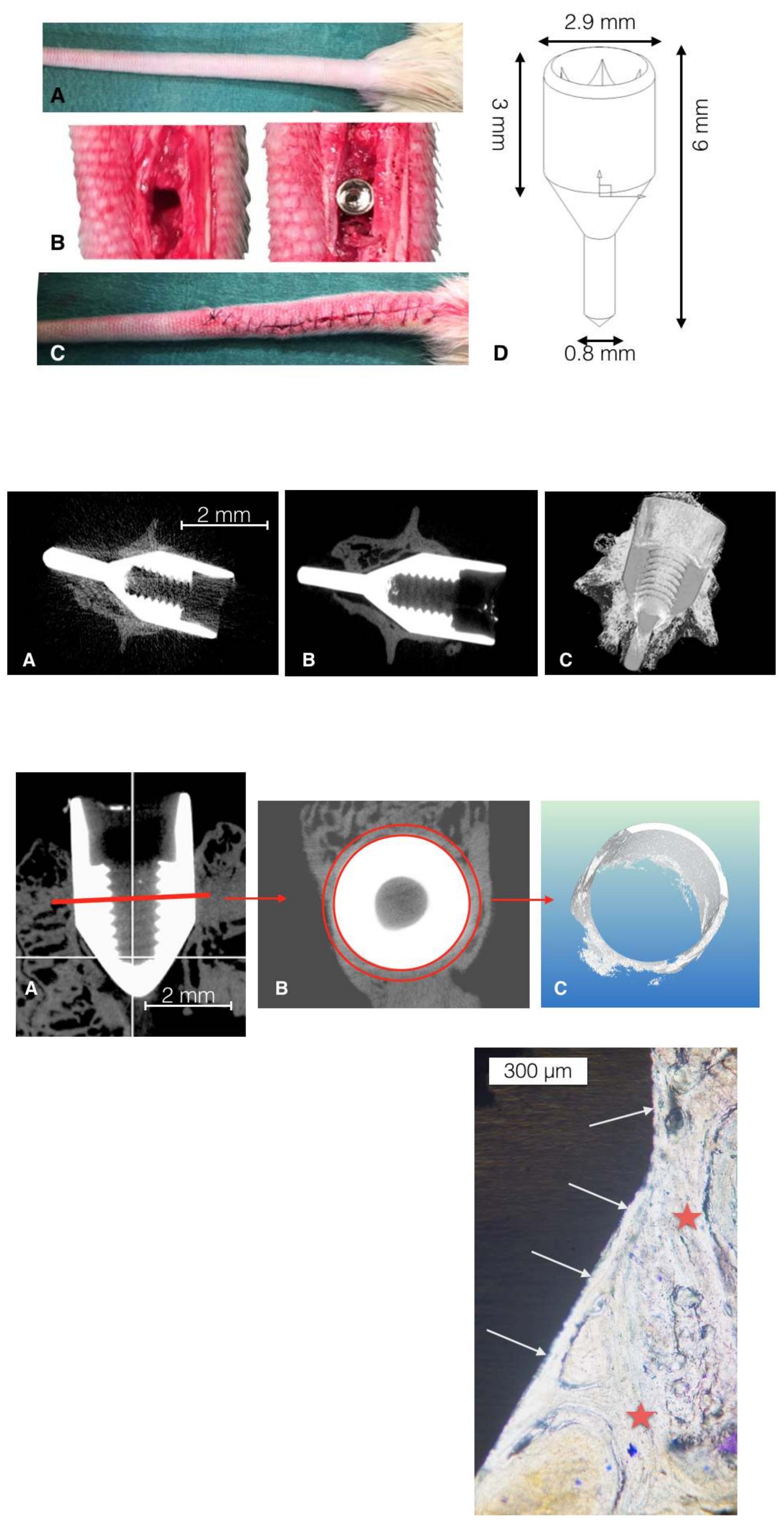

\section{Conclusions}

The rat tail vertebrae model is promising for implant osseointegration studies. The model also permits adapted design implant placements with stability and, without pain and suffers. Micro-CT analysis permits to obtain bone implant contact by bone density calculation. This method can allow building a kinetic using in-vivo Micro-CT and, only on the same animal. For ethical point of view, using this model is also an advantage by decrease the number of animals involved in the study.

\section{References}

Renaud M, Farkasdi S, Pons C, Panayotov I, Collart-Dutilleul PY, Taillades H, Desoutter A, Bousquet $P$, Renaud M, Farkasdi S, Pons C, Panayotov I, Collart-Dutilleul PY, Taillades H, Desoutter A, Bousquet $P$,
Varga $G$, Cuisinier $F$, Yachouh J. "A New Rat Model for Translational Research in Bone Regeneration." Tissue Eng Part C Methods. 2015 Dec 31.

Renaud M, Farkasdi S, Desoutter A, Varga G, Cuisinier F, Bousquet P. "A rat tail model for osseointegration studies and new bone formation follow-up." Journal of Cell Science and Therapy. 2016. 